\title{
A comparison of positive and negative contrast effects
}

\author{
JAMES H. McHOSE and JOHN N. MOORE \\ Southern Illinois University, Carbondale, Illinois 62901
}

\begin{abstract}
Rats originally trained on large reward displayed negative contrast when shifted to a varied large/small reward schedule, while rats trained on the small reward schedule displayed positive contrast when shifted to the varied reward schedule. The durations of the obtained positive and negative contrast effects were comparable. These data, in conjunction with previous findings, suggest an appraisal of the general characteristics of theoretical accounts of contrast effects.
\end{abstract}

It is well known that the behavior of organisms under one reinforcement schedule often depends upon the organism's previous reinforcement history. The contrast effects displayed by rats in response to increases or decreases in the amount of reward received for a particular instrumental response, Crespi's (1942) "elation" and "depression" effects, are examples of such phenomena.

Since Crespi's original report, theoretical overviews of these contrast phenomena have quite naturally mirrored empirical developments. In the nearly three decades following Crespi's work, there were many reports of "depression," or negative contrast effects, while the occurrence of "elation," or positive contrast, was rare in relevant experiments (cf. Black, 1968; Dunham, 1968). Theoretical treatments of the replicable phenomenon, negative contrast, were either inapplicable to the operation of reward increase or predicted the absence of positive contrast. Recently, however, there have been numerous reports of positive contrast attending shifts from small to large reward, and these findings have prompted the revision or extension of the relevant theories of previous decades (cf. Capaldi, in press; McHose \& Moore, 1976). This paper is concerned with the direction such theoretical formulations might take. Central to such concern is some determination of the general nature of the reinforcement variables implicated in contrast effects. Of immediate interest is the question of whether positive and negative contrast effects are essentially symmetric phenomena, for if they are not, it is conceivable that different theoretical processes must be invoked to account for the behavioral effects of reward increase and reward decrease.

One sense in which positive and negative contrast iffects are asymmetric is apparent in the relevant iterature: A given reduction in reward amount may with regularity yield a negative contrast effect, but :omparable increases in reward amount do not yield sositive contrast effects (cf. McHose \& Moore, 1976). I further potentially important difference between the :ffects of reward increase and reward decrease stems from what appears to be the protracted duration, if not permanence, of positive contrast, once obtained (cf. Mellgren, Seybert, Wrather, \& Dyck, 1973), as compared with the typically labile characteristics of the negative contrast effect (e.g., McHose \& Peters, 1975). The experiment reported in this paper explores this apparent discrepancy.

At a purely empirical level, it is possible that the apparent durability of positive contrast effects is attributable, not to any fundamental difference between the effects of reward increases and decreases, but to the level of postshift reinforcement paramenters in the typical positive contrast as compared with typical negative contrast study. Obviously, postshift reinforcer magnitude is typically large in positive contrast studies and small in negative contrast paradigms. In order to compare the durations of positive and negative contrast in the absence of systematic differences between contrast groups (other than the fact that one group experiences reward increase while the other experiences reward decrease), it is necessary to control both the amount of change in reward from preshift to postshift training and the level of postshift reinforcement conditions between groups. Controlling for postshift reward levels between groups is accomplished simply by shifting reward for both positive and negative contrast groups to some common level that is intermediate between the large and small preshift reward values. Selecting an intermediate reward value such that reward-increase and reward-decrease groups experience the same change in reward amount is more complex since the functional difference between two reward amounts is not linearly related to reward amount per se. Stated differently, reward-related constructs such as incentive, $K$, are not assumed to grow linearly with reward parameters (cf. McHose, 1970; Spence, 1960). Equating "amount of change" from preshift to postshift training must be in terms of the $\mathrm{K}$ variable, which is linearly related to behavior, not reward amount. This may reasonably be accomplished by shifting groups to a "random" varied magnitude schedule in which the large and small postshift rewards are equiprobable. Previous data suggest 
that the resultant varied reward schedule is the functional equivalent of a constant magnitude schedule for which the $K$ value is intermediate between the $K$ values corresponding to the smaller and larger reward amounts (McHose \& Peters, 1975).

\section{METHOD}

The subjects were 33 experimentally naive male albino rats, approximately 90 days of age, obtained from the Holtzman Company.

The apparatus was a straight runway described in detail elsewhere (McHose \& Peters, 1975). The runway consisted of start, run, and goal sections with a side-exiting solenoidoperated start door which separated the start and run sections and a guillotine retrace door which separated the run and goal sections. The goal cup was covered by an opaque solenoidoperated lid. Photocell-clock circuitry provided for the measurement of response latencies over three $30-\mathrm{cm}$ segments of the runway, with the first clock starting when the start door was opened. Photocell circuitry also initiated a time-delay cycle when the subject approached the goal cup.

Ten days prior to the first day of training (Day 11), subjects were placed on a 23-h food-deprivation schedule which was maintained throughout the experiment. On Days 1-10 each subject was handled for approximately $5 \mathrm{~min}$. On Days 6-10 each subject was fed $2045-\mathrm{mg}$. Noyes pellets, identical to the subsequent reinforcement pellet, in addition to their scheduled 1-h access to food. On Days 8-10, subjects were given approximately $5 \mathrm{~min}$ access to the start and run sections of the apparatus.

Eleven subjects were randomly assigned to each of three groups, labeled according to the number of pellets received on each preshift trial: Groups 16,16/1, and 1. Group 16/1 received 16 pellets on half of the preshift trials and 1 pellet on half the trials, according to a quasirandom schedule for which the probability of one event (e.g., 1 pellet) on any trial was independent of the preceding trial event. All subjects received this $16 / 1$ schedule of reward during the postshift period. Subjects were administered 30 preshift trials, 1 on Day 11, 2 on Day 12, and 3 trials per day thereafter. There were 75 postshift trials, administered at the rate of 5 per day. All rewards during the preshift and postshift periods were delayed $15 \mathrm{sec}$ from the time the subject entered the goalbox.

Trials were administered to squads composed of eight or nine subjects, with at least two, but no more than three, subjects from each experimental group. Within squads, all subjects received their first daily trial before any subject received its second trial, etc. This procedure resulted in a within-day intertrial interval of approximately 6-9 $\mathrm{min}$.

On each trial, the start door was opened following a 3-sec orientation toward the door; the retrace door was closed and the delay interval initiated when the subject interrupted the last photobeam. At the end of the delay interval, the goal-cup lid retracted, and the subject was removed immediately following consumption of the reinforcement.

\section{RESULTS}

Only the goal measure data will be presented, since this measure proved most sensitive to the manipulation of reward magnitude. Group mean goal speeds, reciprocated latencies, are plotted in Figure 1 as a function of blocks of trials. Performance levels for Groups 16 and 1 are plotted relative to the performance level displayed by Group $16 / 1$ at each trial block. Block A

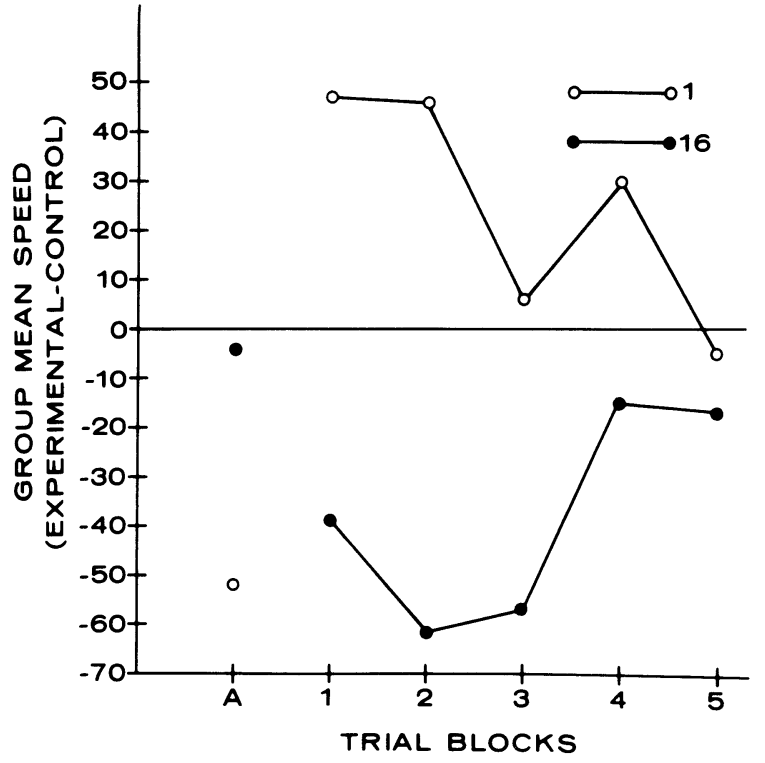

Figure 1. Group mean goal speed difference $(100 / \mathrm{sec})$ for groups at the end of acquisition and over the postshift period. The baseline represents the performance level of Group 16/1.

contains the data for the last 15 trials of the preshift period, while each subsequent block contains the data for successive blocks of 15 postshift trials. Focusing on the postshift data, it may be seen that Group 1 developed faster speeds under the postshift condition of varied (16/1) reward magnitude than did the control group, Group 16/1, which was trained and maintained on the varied reward magnitude schedule. This positive contrast effect diminished over postshift training. Similarly, the group experiencing a reduction in reward magnitude, Group 16, displayed a temporary depression of performance or negative contrast relative to the control group. Analysis of variance of the data for the five trial blocks presented in Figure 1, including blocks and groups as factors, yielded a significant Blocks by Groups interaction $(\mathrm{F}=2.02, \mathrm{df}=8 / 120$. $\mathrm{p}<.05)$. The simple main effect of groups was reliable at both Blocks 1 and $2(\mathrm{Fs}=6.37$ and 10.16, respec, tively, $\mathrm{dfs}=2 / 150, \mathrm{p}<.01)$ and at Block $3(\mathrm{~F}=4.15$ $\mathrm{df}=2 / 150, \quad \mathrm{p}<.05)$. Subsequent pair comparisor. (Tukey) showed that the performance of Group 16 was reliably $(\alpha=.01)$ depressed relative to that of Grour $16 / 1$ at Blocks 1,2 , and 3, while the performance of Group 1 was elevated relative to that of Group 16/1 a1 Blocks 1 and 2.

\section{DISCUSSION}

The present data suggest that the duration of a contras effect produced by a given change in incentive is essentiall! independent of the direction of that change when postshif reinforcement level is controlled between reward-increase anc reward-decrease groups. (In the present data negative contrast if anything, lasted longer than did the obtained positive contrast although an inference of any generality to this conclusior 
appears ill-advised in view of the seemingly spurious decrease in the extent of positive contrast at Block 3.) Previous data suggest that positive and negative contrast effects are similarly affected by reward-delay/reward-amount interactions (cf. Moore $\&$ McHose, 1975) and by trial-to-trial variations in reward amount (e.g., Lehr, 1974; McHose \& Peters, 1975). These data, in conjunction with the present findings, yield an empirical summary to the effect that there appears to be no current basis for the assumption that reward increase and reward decrease affect behavior through different processes.

The minimum paradigmatic design required for the isolation of variables producing contrast effects is a simple 2 by 2 factorial manipulation of some preshift and postshift reward condition, such as reward amount. If symmetric positive and negative contrast effects occur following the operations of reward increase and reward reduction, one, and only one, variable has been identified. That variable is the absolute value of the preshift reward. In the language of the factorial, a main effect of preshift reward is obtained such that the smaller the training (preshift) reward, the higher the level of performance when reinforcement conditions are equated between comparison groups. Reward increase and decrease are but two different procedures for equating reinforcement conditions between groups, that is, two different procedures for observing the effect of one variable, preshift magnitude. Whatever form theoretical treatments of contrast may take, the isolation of preshift magnitude as a variable responsible for contrast cautions against the introduction of one theoretical process to account for positive contrast and another to account for negative contrast. Such theoretical approaches are as old as Crespi's (1942) original analysis, in which reward decrease was assumed to activate some negative response-debilitating affective state; reward increase presumably activated some positive response-facilitating affective state. Capaldi's (in press) reinforcement level theory, in which reward decrease creates inhibition and reward increase provides for supernormal habit growth, is a contemporary version of this sort of approach, although the theoretical processes introduced to account for the effects of reward increase and reward decrease are "associative" rather than "motivational" in character.

While positive and negative contrast are in some respects symmetric phenomena, there is at least one important respect in which the two phenomena are not symmetric. In any survey of the relevant literature (cf. Black, 1968; Dunham, 1968; McHose \& Moore, 1976), it is clear that specific changes in reward amount more often produce negative than positive contrast. That is, negative contrast is the more robust and ubiquitous phenomenon. Again referring to the 2 by 2 factorial, it would appear that preshift and postshift reward amounts interact such that change in reinforcement schedule debilitates performance. Indeed, it is this variable that is interpreted within the Capaldi and Lynch (1967) analysis of contrast and implicated in the approach we have adopted elsewhere (McHose \& Moore, 1976). Both approaches interpret this variable in terms of stimulus change (generalization decrement). Thus, negative contrast is attributed either wholly (Capaldi \& Lynch, 1967) or in part (McHose \& Moore, 1976) to a change, from the preshift to the postshift periods, in the value of the reward-produced stimuli to which response was initially conditioned. Note that the reward-change variable (preshift by postshift reward magnitude interaction) will enhance the amount of negative contrast and reduce the amount of positive contrast to be expected from the operation of the preshift reward magnitude variable. That $s$, the two experimental variables act in concert to yield large regative effects, while the variables act in opposition when eward is increased, suggesting some difficulty in observing ositive contrast following reward increase.

While two variables are implicated in the occurrence of ontrast, the third variable in the conceptual factorial, postshift eward magnitude, appears to influence the duration of contrast, ince obtained. When postshift reward levels are equated between reward-increase and reward-decrease groups, as in the present experiment, positive and negative contrast effects are of approximately equal duration. However, when post shift reward level is high, (positive) contrast appears extended in duration relative to the course of (negative) contrast when postshift reward level is low.

Recognition of the two variables, preshift reward level and change in reward level, as responsible for positive and negative contrast effects is a helpful antecedent to a consideration of the theoretical explanation of these effects. Given the isolation of these variables as determinants of contrast, at least two conclusions concerning the number of explanatory constructs that should be introduced to account for the phenomena of contrast seem warranted. First, as previously suggested, any relevant theoretical description is overdetermined, lacks parsimony, to the extent that two separate sets of constructs are invoked to account for positive and negative contrast effects. Such a theoretical approach is analogous to the introduction of one set of constructs to account for a partial reinforcement effect when all rewards are large and of another set to account for a partial reinforcement effect when all rewards are small.

A second conclusion also stems from an adherence to the principle of parsimonious theoretical description. Theoretical treatments of the learning process have, for reasons beyond the scope of this discussion, frequently entailed the assumption that stimulus events identified as reinforcers also have the potential cue or discriminative stimulus properties assigned to all stimuli (e.g., Amsel, 1967; Capaldi, 1967; Spence, 1960). Given such an assumption, parsimony is served to the extent that behavioral phenomena associated with manipulations of the reinforcement event are interpreted with theoretical concepts applicable to all stimuli, as opposed to those (e.g., frustration, elation, inhibition) that are reinforcer specific. The "habit generalization" model so successfully developed by Capaldi (1967) provides an excellent example of an interpretation of the effects of the rewardchange variable, which is devoid of reinforcer-specific constructs. In a similar vein, the effects of the preshift magnitude variable are interpretable as consequences of the stimulus (cue) properties of reinforcement events without appeal to reinforcer-specific explanatory mechanisms (cf. McHose \& Moore, 1976).

\section{REFERENCES}

Amsel, A. Partial reinforcement effects on vigor and persistence. In K. W. Spence \& J. T. Spence (Eds.), The psychology of learning and motivation. New York: Academic Press, 1967.

BlACK, R. W. Shifts in magnitude of reward and contrast effects in instrumental and selective learning: A reinterpretation. Psychological Review, 1968, 75, 114-126.

CAPALDI, E. J. A sequential hypothesis of instrumental learning. In K. W. Spence \& J. T. Spence (Eds.), The psychology of learning and motivation (Vol. 1). New York: Academic Press, 1967.

CAPALDI, E. J. Reinforcement level: An expectancy-associative approach to relative reinforcement and nonreinforcement effects. In J. W. Baerwaldt (Ed.), Arlington symposium on learning. Stamford, Conn: Greylock, in press.

Capaldi, E. J., \& LYNCH, D. Repeated shifts in reward magnitude: Evidence in favor of an associational and absolute (noncontextual) interpretation. Journal of Comparative and Physiological Psychology, 1967, 75, 226-235.

CrespI, L. P. Quantitative variation of incentive and performance in the white rat. American Journal of Psychology, 1942, 55, 467-517.

Dunham, P. J. Contrasted conditions of reinforcement: A selected critique. Psychological Bulletin, 1968, 69, 295-315.

LEHR. R. Partial reward and positive contrast effects. Animal Learning \& Behavior, 1974, 2, 221-224. 
McHose, J. H. Relative reinforcement effects: $S_{1} / S_{2}$ and $S_{1} / S_{1}$ paradigms in instrumental conditioning. Psychological Review, 1970, 77, 135-146.

McHose, J. H., \& Moore, J. N. Expectancy, salience, and habit: A noncontextual interpretation of the effects of changes in the conditions of reinforcement on simple instrumental responses. Psychological Review, 1976, 83, 292-307.

Mchose, J. H., \& Peters, D. P. Partial reward, the negative contrast effect, and incentive averaging. Animal Learning \& Behavior, 1975, 3, 239-244.

Mellgren, R. L., Seybert, J. A., Wrather, D. M., \& Dyck, D. G. Preshift reward magnitude and positive contrast in the rat. American Journal of Psychology, 1973 , 86. $383-387$.

Moore, J. N., \& McHose, J. H. The effects of delay of reward on negative contrast effects associated with reductions in reward magnitude. Bulletin of the Psychonomic Society, 1975, 6, 497-500.

SPENCE, K. W. Behavior theory and learning. Englewood Cliffs, N.J: Prentice-Hall, 1960.

(Received for publication February 9, 1978.) 